\title{
Germanica
}

\section{Du roman américain sur le nazisme à la BD allemande : transferts, adaptations et jeux d'intermédialité - à propos de Die Welle. Eine Graphic Novel de Stefani Kampmann}

Vom amerikanischen Roman über den Nationalsozialismus zum deutschen

Comic : Transfers, Variationen und Intermedialität - Über Die Welle. Eine graphic Novel von Stefani Kampmann

From an American novel on Nazism to German comics book : transfers, variations and intermediality - about Die Welle. Eine graphic Novel by Stefani Kampmann.

\section{Claire Aslangul}

\section{OpenEdition}

Journals

\section{Édition électronique}

URL : http://journals.openedition.org/germanica/1168

DOI : 10.4000/germanica. 1168

ISSN : 2107-0784

\section{Éditeur}

Université de Lille

\section{Édition imprimée}

Date de publication : 31 décembre 2010

Pagination : 161-176

ISBN : 9782913857261

ISSN : 0984-2632

Référence électronique

Claire Aslangul, «Du roman américain sur le nazisme à la BD allemande : transferts, adaptations et jeux d'intermédialité - à propos de Die Welle. Eine Graphic Novel de Stefani Kampmann », Germanica [En ligne], 47 | 2010, document 10, mis en ligne le 01 décembre 2012, consulté le 06 octobre 2020. URL : http://journals.openedition.org/germanica/1168; DOI : https://doi.org/10.4000/germanica.1168

Ce document a été généré automatiquement le 6 octobre 2020.

(c) Tous droits réservés 


\section{Du roman américain sur le nazisme à la BD allemande : transferts, adaptations et jeux d'intermédialité - à propos de Die Welle. Eine Graphic Novel de Stefani Kampmann}

Vom amerikanischen Roman über den Nationalsozialismus zum deutschen Comic : Transfers, Variationen und Intermedialität - Über Die Welle. Eine graphic Novel von Stefani Kampmann

From an American novel on Nazism to German comics book : transfers, variations and intermediality - about Die Welle. Eine graphic Novel by Stefani Kampmann.

Claire Aslangul

1 «Vous pensez donc qu'une dictature serait chez nous impossible ? $»^{1}$ Pourtant, n'y a-til pas au fond de chaque être humain - a fortiori chez ceux qui souffrent de diverses frustrations - une tendance viscérale à suivre un chef et un besoin instinctif de se sentir appartenir à une communauté ? En soulevant ces questions dans le pays qui a vu naître le nazisme, le film de Dennis Gansel Die Welle, sorti en $2008^{2}$, a fortement attiré l'attention des médias. Actualisant un roman américain de la fin des années 1970, il transpose dans l'Allemagne d'aujourd'hui une histoire que les lycéens allemands connaissent bien : Die Welle, dans sa traduction disponible depuis 1984, est en effet pour eux depuis de nombreuses années une lecture quasi obligatoire. La publication presque concomitante (2007) du " roman graphique » (Graphic Novel) de Stefani Kampmann ${ }^{3}$ sur le même thème est passée plus inaperçue. Ce très bel ouvrage en noir et blanc soulève pourtant d'intéressantes questions.

2 La démarche des différentes versions de Die Welle étant ouvertement éducative, cette variation sur le sujet invite d'abord à réfléchir sur le statut de la bande dessinée 
aujourd'hui. Car longtemps décrié et soupçonné de corrompre la jeunesse, on voit ici le genre réquisitionné au service d'une cause d'une grande importance, particulièrement en Allemagne: le regard critique et éclairé sur l'héritage national-socialiste. Il faut aussi s'interroger sur les moyens mis en œuvre pour adapter « efficacement » le roman en bande dessinée : comment l'auteure s'y prend-elle pour rendre les atmosphères et émotions lorsque le texte se fait moins abondant mais a l'avantage d'être secondé par l'image? Qu'advient-il de la narration originelle? Est-elle respectée, simplifiée, complétée?

3 Si c'est bien ici la bande dessinée qui nous intéresse, ces problématiques prennent un relief particulier lorsqu'on établit des comparaisons entre le support BD et le support cinématographique, lequel mélange lui aussi texte (parlé) et image. La mise en relation des deux types de médias est d'autant plus pertinente dans ce cas précis que le temps de lecture / visionnage des deux œuvres est similaire (environ deux heures) et que le public visé est le même. Les formes BD et cinéma impliquent des choix de dramatisation que l'on pourrait penser proches l'un de l'autre et qui sont en réalité très différents. Ces écarts aident à percevoir la spécificité de chaque média et permettent d'envisager aussi les différences de pratique dont ils font l'objet.

4 Les adaptations en images du roman de Rhue renvoient par ailleurs à des questionnements d'ordre sociologique : le roman initial se déroulait dans l'Amérique des années 1960-70, et avait été écrit pour le public américain de l'époque ; le film et la bande dessinée sont des productions allemandes, destinées à un public d'aujourd'hui. Quelles en sont les conséquences? Quelles transformations ont subi l'histoire et les personnages en traversant le temps et l'Atlantique? Les choix du cinéaste et ceux de l'illustratrice de bande dessinée sont divergents : cela tient-il aux particularités des supports choisis? Quels autres facteurs entrent en ligne de compte - les impératifs commerciaux, les «cahiers des charges» imposés par les commanditaires, les contraintes techniques?

5 Nous retracerons ici l'histoire de l'objet qui nous intéresse, la bande dessinée de Kampmann et son contexte de réalisation, avant de nous pencher sur les enjeux de l'adaptation, dans un genre dit "mineur", d'un sujet difficile. Nous verrons ensuite comment, à la recherche d'un juste équilibre entre émotion et raison, l'auteure a exploité les potentialités du medium bien particulier qu'est la bande dessinée.

\section{The Wave / Die Welle - de la réalité aux différents supports de la fiction, 1967-2008}

6 La circulation dans les différents médias, époques et pays de l'expérience Die Welle mériterait à elle seule un article, et le bref résumé que nous proposons ici ressemble quelque peu à un jeu de poupées russes. Il permet de mettre en évidence les buts communs qui animent le réalisateur initial de "l'expérience» et tous ceux qui, écrivains, cinéastes, dessinateurs, ont ensuite utilisé l'idée initiale dans leurs productions; mais ce rapide historique révèle aussi d'importantes différences de conception liées notamment à l'évolution des contextes de production.

7 L'expérience originelle, menée en 1967 par Ron Jones, professeur d'histoire à la Cubberly Highschool de Californie ${ }^{4}$, est ancrée dans une époque où les questions de dynamique de groupe et de rapport entre l'individu et le pouvoir travaillent fortement 
la sociologie américaine. Il n'est que de citer la célèbre expérience de Milgram ${ }^{5}$ pour se remémorer à quel point, dans la recherche outre-atlantique, le questionnement sur les phénomènes dictatoriaux va alors de pair avec une interrogation sur un phénomène supposé universel : l'obéissance à l'autorité. C'est donc bien loin de l'Allemagne, mais dans une génération d'intellectuels proches encore, chronologiquement, de l'ère nationale-socialiste, que naît l'initiative de Jones: suite à la réflexion d'un élève, incrédule quant à la possibilité que les Allemands n'aient rien su de l'Holocauste, et catégorique sur le fait qu'un régime comme celui du Troisième Reich ne pourrait jamais plus voir le jour, le professeur teste la docilité du groupe qu'il a en face de lui. Taraudé par la question "le désir de discipline et d'uniformité est-il donc un besoin naturel ? $»^{6}$, l'enseignant propose de nouvelles règles, instaure de nouveaux principes - la discipline, la communauté - auxquels, à sa grande surprise, les élèves se rallient avec entrain. Un salut, des cartes de membres, une façon particulière de s'habiller viennent bientôt renforcer la cohésion de la classe et le « mouvement » s'autonomise, échappe à son initiateur ; l'enseignant, qui s'est lui aussi pris au jeu, interrompt l'expérience après avoir constaté les dérapages de la classe et, notamment, le potentiel d'exclusion et de violence inhérent au processus de formation d'un groupe discipliné. Ayant demandé à ses élèves de garder l'expérience secrète, Jones revient lui-même en 1972, après plusieurs années de silence, sur cet épisode qui l'a énormément marqué, dans un récit intitulé The Third Wave.

Le caractère très probablement fictionnel de certains éléments relatés par le professeur n'a pas constitué une entrave au succès de l'histoire, particulièrement en Allemagne, dans le contexte d'un premier « retour de mémoire » qui a vu notamment la diffusion de la série Holocaust à la télévision (en 1978). C'est pour ce même média que le récit de Jones est adapté en 1981 par Alexander Grasshoff7. Mais l'essor véritable de Die Welle commence lorsque Morton Rhue (alias Tod Strasser), journaliste et essayiste américain, écrit d'après le scénario du film de Grasshoff son roman The Wave. Publié en 1981 aux États-Unis, il est traduit en allemand en 1984 et paraît chez Ravensburger. Son succès ne s'est pas démenti depuis.

Il est envisageable de ne voir qu'une simple coïncidence dans l'adaptation en bande dessinée et la sortie d'un nouveau film sur Die Welle en 2007-2008. Les deux productions sont très différentes l'une de l'autre, nous y reviendrons, et Stefani Kampmann affirme n'avoir rien su de la préparation du film tandis qu'elle travaillait à sa bande dessinée ${ }^{8}$. Sollicités sur le sujet, les responsables de Ravensburger Verlag, qui ont publié également en 2008 un roman d'après le scénario du nouveau film ${ }^{9}(!)$, n'ont pas répondu à nos questions sur une éventuelle stratégie commerciale d'ensemble. On peut néanmoins constater que chaque support - roman de Rhue, roman de Winter d'après le nouveau film, DVD du film de Gansel, BD de Kampmann - fait de la publicité pour les autres productions, déclinant ainsi La Vague selon le principe des "produits dérivés ". Un certain nombre d'observateurs l'a d'ailleurs fait remarquer en usant de métaphores faciles : "Après Die Welle, adapté du roman de Todd Strasser, La Vague se décline en version dessinée. Est-on emporté par le tsunami ou pataugeons-nous dans une petite flaque $? »^{10}$. Le succès renouvelé des différents avatars de La Vague est donc sans conteste en partie dû à une stratégie marketing (plus ou moins planifiée).

Il révèle surtout, indéniablement, l'existence d'un marché particulièrement porteur : celui de la mémoire, extrêmement florissant, que l'on s'en offusque comme d'un signe de «marchandisation » de l'histoire et symptôme de banalisation de l'Holocauste, ou 
que l'on s'en réjouisse comme preuve que l'oubli n'a pas submergé les jeunes générations. Indiscutablement, la période actuelle, qui voit la disparition des derniers témoins vivants, est une période-clé, pour laquelle le recul manque encore mais qui fait apparaître l'existence d'un véritable «boom de la mémoire $»^{11}$. Il n'est d'ailleurs sans doute pas anodin que Kampmann et Gansel, nés tous deux en 1973, appartiennent à une génération bien particulière - celle " qui n'a jamais connu la guerre $~^{12}$ mais qui a vu les derniers témoins; celle qui est peut-être "ni trop près ni trop éloigné(e) $»^{13}$ de la période concernée, et éminemment consciente de se situer à la charnière avec une génération différente.

11 Il s'agissait pour Jones ou Rhue, il y a plusieurs dizaines d'années, de briser un tabou (avec une réflexion sur le potentiel totalitaire des individus à l'intérieur même de l'État démocratique nord-américain) et d'évoquer une période encore largement méconnue des écoliers; l'enjeu est tout autre pour les auteurs allemands d'aujourd'hui : ils se trouvent confrontés à un public qui a plutôt trop d'informations que pas assez, et qu'il faut d'autant plus toucher par l'émotion et la fiction que les victimes réelles disparaissent.

Quelles qu'en soient les modalités de mise en forme, l'histoire de Die Welle, importée des États-Unis, s'intègre de manière particulièrement pertinente dans la pédagogie de la mémoire telle qu'elle est pratiquée outre-Rhin ces dernières décennies: Alexandra Oeser, dans un ouvrage publié récemment ${ }^{14}$, a démontré à quel point l'enseignement du nazisme était, dans les écoles allemandes, basé sur l'empathie (ce qu'elle appelle la Betroffenheit). Il s'agit d'inciter les élèves à s'interroger personnellement sur la manière dont eux-mêmes se seraient comportés sous le régime nazi, en s'appuyant sur une démarche d'identification aux victimes. Or Die Welle propose un type d'identification spécifique : on a en effet affaire ici à un processus d'identification non pas aux victimes, mais bien aux bourreaux - les jeunes qui lisent la bande dessinée se projettent dans les figures d'adolescents du même âge qui sont emportés par La Vague; victimes consentantes de leur professeur-apprenti dictateur, ils sont surtout complices et auteurs eux-mêmes de formes exacerbées d'exclusion de toute personne réputée "différente » et n'adhérant pas à la "cause ». Ce choix risqué de l'identification aux tortionnaires implique, de la part des auteurs, qu'à un moment ou un autre soit exprimée clairement l'idée que ce qui est raconté est le contraire de ce qu'il fallait faire. C'est une des raisons pour lesquelles dans le roman de Rhue, le film et la bande dessinée (mais en contrepoint du récit de Ron Jones), des figures "positives» de résistant(e)s sont fortement mis en valeur, afin d'éviter toute ambiguïté. C'est aussi ce qui explique que Gansel ait modifié la fin, pour signaler que la séduction de la communauté devait être démasquée et créer ainsi un véritable repoussoir.

\section{Évoquer le nazisme en BD aujourd'hui : quels risques, quels atouts, quelles stratégies de légitimation pour un « genre mineur " ?}

13 L'attrait de la discipline et la tentation de la dictature constituent des risques inhérents à l'histoire même de Die Welle, quel que soit le mode du récit. Entreprendre d'adapter cette histoire en bande dessinée, c'est de surcroît s'exposer au reproche d'une possible 
«trivialisation» du sujet. La bande dessinée n'est en effet pas réputée un genre " sérieux »15, et le traitement d'un thème comme l'Holocauste peut paraître délicat :

Si les comic books sont assez largement considérés comme une littérature mineure, légère et populaire, que dire des prétentions d'une bande dessinée qui traite d'un thème aussi grave que celui de la Shoah ? (...) Dans un article qu'il consacre à la littérature de l'Holocauste, Robert S. Levanthal note que les critiques ont été nombreux à considérer qu'on ne pouvait parler du Génocide que dans des genres élevés, c'est-à-dire les genres nobles de la littérature ${ }^{16}$.

Certes, de grands auteurs reconnus se sont attaqués au sujet sans que leur soit contestée une forte légitimité - il n'est que de citer le fameux Maus d'Art Spiegelmann. Certes aussi, la BD «historique » et sérieuse a acquis ces dernières années quelques lettres de noblesse ${ }^{17}$ et la Geschichte im Comic est en passe de se voir reconnue comme genre à part entière ${ }^{18}$. Néanmoins, le support même reste suspect, d'autant plus lorsque comme ici, le graphisme situe ostensiblement l'œuvre dans la catégorie «manga ». Il est intéressant de constater que même des " jeunes ", le public par excellence du roman graphique de Kampmann, émettent des réserves quant à la pertinence de ce média, ainsi que l'atteste cette présentation de l'ouvrage à l'occasion de la Buchmesse de 2009 :

Jeremy Schmidt (13 ans) et Helena Stiller (14 ans) présentent :

Rhue, Kampmann « La Vague » (Roman graphique)

Le livre est facile à lire, parce que les dessins sont bien faits. (...) Mais à notre avis, la thématique sérieuse du livre ne devrait pas être traitée sous forme de bande dessinée ${ }^{19}$.

Dans leurs critiques de l'ouvrage, les observateurs mettent systématiquement l'accent sur le risque de banalisation (Verharmlosung) que semble impliquer le genre BD, même si c'est pour souligner immédiatement que Stefani Kampmann a su relever le défiavec des formulations similaires à celles-ci :

Adapter en bande dessinée le classique de la littérature de jeunesse de Morton Rhue La Vague, qui traite de manière critique de la naissance du nazisme et de l'extermination des Juifs - cela ne conduit-il pas immanquablement à euphémiser / banaliser un sujet éminemment délicat? La dessinatrice berlinoise Stefani Kampmann nous montre le contraire : dans son roman graphique novateur (...), elle développe son propre langage visuel de l'horreur ${ }^{20}$.

Quoique d'abord méfiants, les critiques s'accordent donc sur la réussite d'une entreprise difficile. Presque tous reconnaissent même que la bande dessinée apporte un "plus» par rapport au roman, avec une remarque récurrente: le caractère «accrocheur» du medium, la lecture aisée permettent une grande diffusion de l'histoire et une bonne compréhension, bien mieux que ne le ferait un roman. Un critique note ainsi :

L'histoire, prenante, est mise en images de manière divertissante et sérieuse en même temps, et le contenu est réduit à l'essentiel sans que rien soit enjolivé. Le medium de la bande dessinée est de plus idéal pour ceux qui ont la paresse de lire et il donne envie d'aller plus loin ${ }^{21}$.

Dans un processus de retournement tout à fait intéressant, c'est précisément ce l'on reprochait à la bande dessinée qui devient un atout reconnu pour la réflexion sur le nazisme chez un lectorat jeune réputé peu assidu : une nouvelle enveloppe, dynamique et allégée en texte, pour un classique, cela ne constitue pas une dénaturation de l'œuvre initiale, ni ne conduit à la «trivialisation» d'un sujet douloureux, mais au contraire, cela ouvre la perspective de gagner de nombreux lecteurs : 
Un classique dans une forme complètement nouvelle, pour sûr, tout le monde n'y trouvera pas son compte, mais pour les jeunes, c'est en tout cas quelque chose qui parle - en particulier en cette époque des dessins animés et des mangas ${ }^{22}$. sujet du livre, explique :

L'éditeur m'a demandé si j'aimerais adapter La Vague sous forme de roman graphique. Je ne connaissais le livre que par ouï-dire mais en le lisant, j'ai vite remarqué que le thème était passionnant et surtout fondamental. Aujourd'hui encore, il y a des accès de violence contre des minorités. Les groupes d'extrême droite sont toujours très actifs et tentent de séduire les jeunes. Il faut faire quelque chose contre ça. Par ailleurs, je pense qu'il est important que les jeunes se confrontent avec la dynamique de groupe et les pressions qui peuvent en découler. Si ce livre, grâce à la forme de la bande dessinée, peut toucher encore plus de lecteurs, alors c'est tant mieux ${ }^{23}$. n'allant pas de soi, on notera que l'auteure a mis en œuvre toute une série de procédés de légitimation - qui ont visiblement convaincu la critique en installant la crédibilité éducative de l'ouvrage.

Le choix du noir et blanc fait partie de ces procédés au premier chef : il permet d'éviter l'écueil de l'esthétisation et d'ancrer l'œuvre dans la filiation d'autres bandes dessinées reconnues, notamment le Maus de Spiegelmann déjà évoqué (et ce même si Stefani Kampmann, interrogée sur le sujet, n'évoque pas le dessinateur parmi ses sources d'inspiration). Certes, selon les dires de l'auteure, le renoncement à la couleur faisait partie du cahier des charges de l'éditeur; néanmoins, Kampmann semble adhérer profondément à ce qui était initialement un impératif d'ordre technique, car pour elle, " le noir et blanc est sérieux, il réduit à l'essentiel et convient à cette thématique difficile $»^{24}$.

21 Parmi les autres éléments d'une stratégie qui vise à justifier la pertinence de l'entreprise, on attendrait de manière assez naturelle une référence à la " réalité » des faits relatés, en guise de caution. Or, tandis que le roman de Rhue comme le film de Gansel invoquent Ron Jones et la véracité historique de l'expérience Die Welle comme instances légitimantes ${ }^{25}$, on ne trouve rien de similaire dans la BD de Stefani Kampmann. Aucune formule-type comme "d'après une histoire vraie " " nach einer wahren Begebenheit ") ne vient apporter de garantie " externe "; Kampmann se libère donc de ce que Cristina Nord, critique de la taz, a dénoncé comme la "nouvelle forme de naïveté » des récits cinématographiques contemporains à teneur historique, qui consiste, pour convaincre le spectateur, à se parer des atours de l'authenticité, devenue nouveau "fétiche »- comme si la supposée authenticité suffisait à garantir la qualité d'une production ${ }^{26}$.

La «vérité historique » est pourtant convoquée de manière extrêmement forte dans l'ouvrage de Kampmann, et ce par l'insertion d'images authentiques de l'Holocauste lui-même. (ill. 1) 
III. 1, p. 14-15
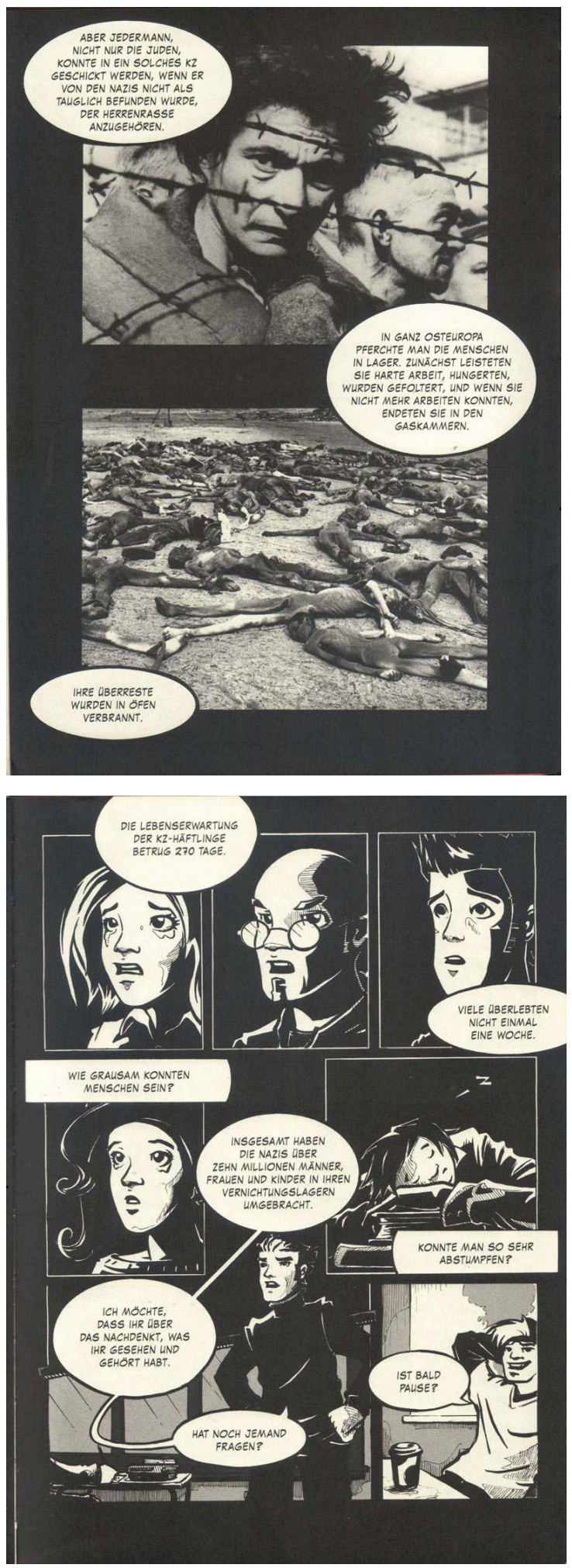

Les photographies, avec leur effet de réel - le ça-a-été de Barthes ${ }^{27}$ - prennent un relief particulier et contrastent fortement au milieu des dessins, introduisant un décalage, un choc plus fort par exemple que lorsque des images filmiques de l'Holocauste sont insérées dans un autre récit filmique, ou que lorsqu'à la place de photogrammes, on a dans la BD des dessins d'après photographies ${ }^{28}$. Ici, au lieu de la description que le 
professeur, dans le roman, fait à ses élèves de la réalité nazie, on trouve, par la mise en abyme (le recours à l'image dans l'image), une convocation forte des événements de l'époque. Si cela contribue à ancrer l'œuvre dans le champ des productions " sérieuses ", c'est aussi très efficace sur le plan émotionnel.

\section{Émotion, condensation, réorganisation et exploitation des potentialités du medium BD}

L'émotion est d'une manière générale un ressort important de l'ouvrage de Kampmann. L'auteure y a recours néanmoins sans dramatiser la trame du récit - à la différence de ce que propose le film de Gansel qui, sur ce point, a d'ailleurs été assez controverséé Sans se démarquer, dans l'intention, des propos du réalisateur qui prône un « rapport plus émotionnel à l'histoire $\aleph^{30}$ pour élargir le public et le captiver, Stephani Kampmann a fait de son côté le choix de la fidélité au texte originel. Son actualisation du récit de Rhue fait néanmoins la part belle à l'affect, en exploitant les potentialités spécifiques du support, en particulier dans la représentation des physionomies humaines; la succession des émotions qui submergent David lors de sa dispute avec Laurie (p. 127) est ainsi rendue très précisément par trois cases juxtaposées (ill. 2). La virulence du dialogue de Laurie avec son petit ami p. 91 est signifiée par la succession rapide de cases présentant alternativement l'un et l'autre des protagonistes, avec l'utilisation d'un lettrage spécifique pour exprimer que le ton monte, et une diagonale finale indiquant le crescendo de l'échange (ill. 3).

ill. 2

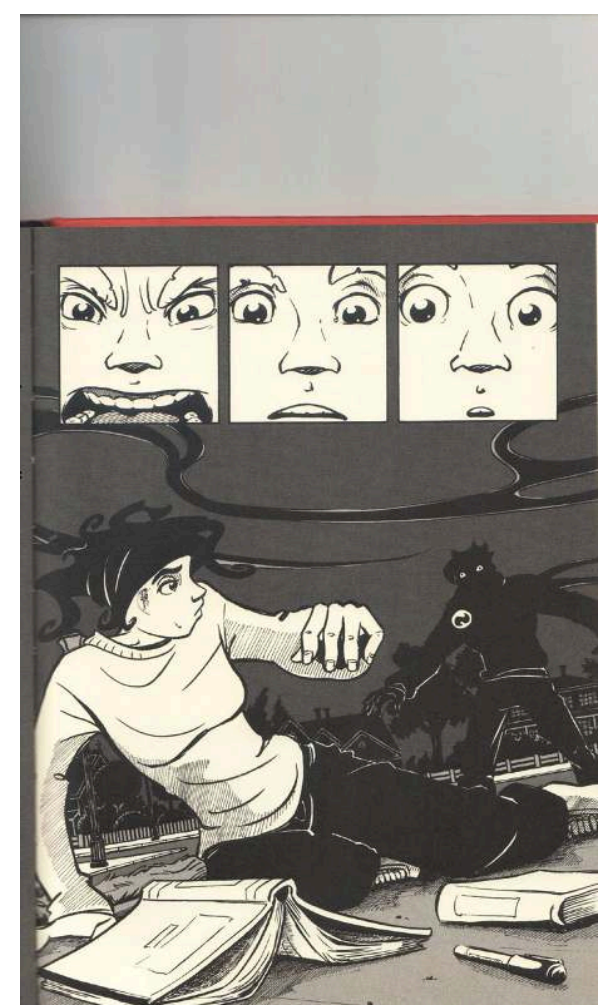


ill. 3

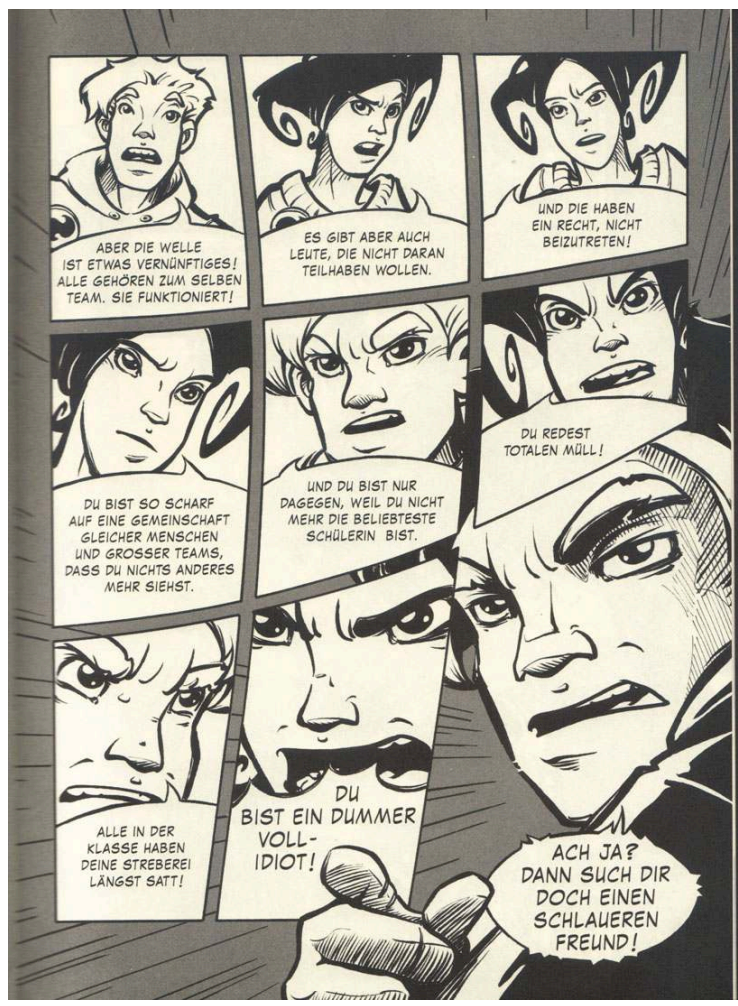

L'usage parcimonieux des images en pleine page permet à l'auteure de donner à ses quelques rares cases-planches une grande expressivité (voir par exemple p. 53, ill.4). Par ailleurs, la représentation du mouvement, qui situe le graphisme dans la filiation d'un univers manga propice à capter l'attention du public, la typographie différenciée pour marquer le ton des échanges, la présence de bulles rondes pour les dialogues et de bulles carrées pour les réflexions intimes des personnages créent des effets tout à fait prenants.

ill. 4 


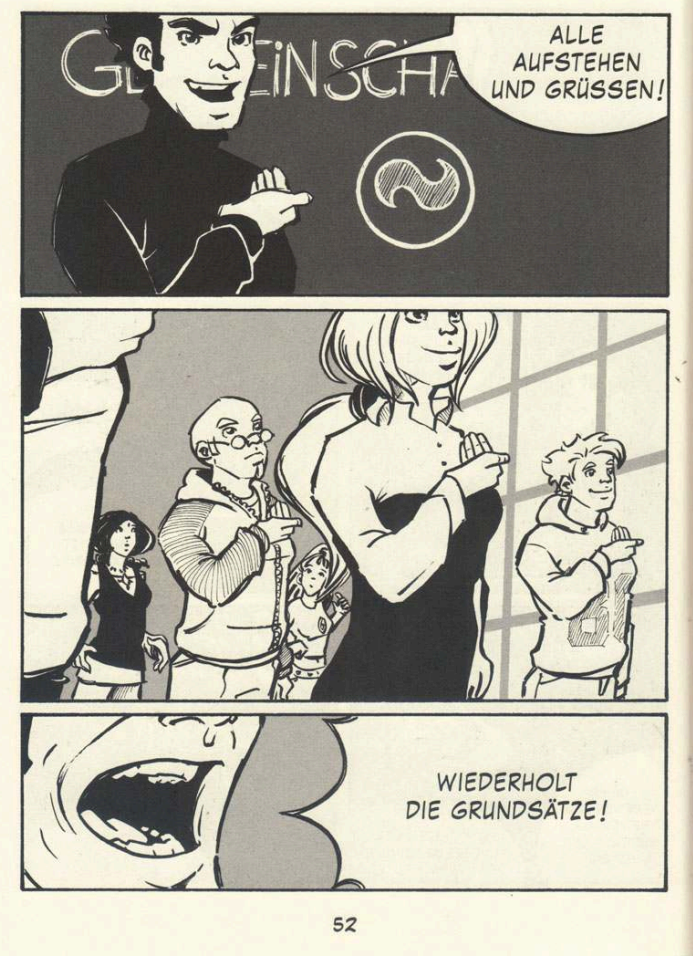
protagonistes, au moment de la perception que le lecteur a des cases d'une planche (on parle de "solidarité iconique »), se révèle particulièrement utile dans une histoire où il est question de l'interaction entre un professeur et une classe. Ainsi, tandis que le romancier raconte d'abord le contenu du discours de l'enseignant, puis la réaction des élèves, l'auteur de bande dessinée peut, lui, faire cohabiter sur la même page ces deux moments, perçus d'un même mouvement par le lecteur. Cela donne des images très fortes, comme celles des p. 52-53 (ill.4) ; ici, on note de manière frappante, entre la page de gauche et la page de droite, l'effet de miroir entre le professeur et ses élèves, avec la mise en image du processus d'action/réaction.

La possibilité de proposer au regard deux visions, deux points de vue d'un même moment, induit de manière générale une perception très différente de celle qui est suscitée par la lecture du roman. Lorsque Morton Rhue use de l'artifice langagier «pendant ce temps» comme expédient pour signifier la simultanéité de deux actions nécessairement narrées successivement, Kampmann peut tout à fait, pour sa part, représenter cette simultanéité. On en trouve un bon exemple lorsque Rhue, p. 173 dans le roman, nous explique que «pendant le discours final du professeur, David et Laurie cherchent à entrer dans la salle, mais se heurtent à plusieurs portes closes "; dans la BD - ce qui serait aussi impossible au cinéma, sauf à couper l'écran en deux - on voit en même temps ce qui se passe au-dedans et au-dehors de la salle, car les vignettes sont réunies sur la même page (p. 154-155, ill. 5). L'effet dramatique est puissant, et sans aucun doute plus apte à captiver de jeunes lecteurs que la lecture des descriptions successives de deux lieux et deux actions pour un même moment; le regard qui embrasse la page permet l'économie d'un effort de synthèse évident.

Parmi les techniques qui contribuent à rendre le récit en images éminemment efficace et émotionnellement «accrocheur », il convient de noter l'habile jeu sur les points de 
vue auquel se livre l'auteure. Si, comme le cinéaste Gansel, elle a souhaité renforcer l'identification du jeune public avec les personnages, Stefani Kampmann n'a pas opté pour une transposition de l'histoire dans l'Allemagne d'aujourd'hui, mais a préféré une mise en scène originale des perspectives : le narrateur omniscient du roman cède ainsi la place à deux perspectives internes, celle de Laurie d'une part (la figure féminine résistante) et celle du professeur. Voici comment l'auteure justifie cette démarche: ill. 5

Grâce à cette narration à la première personne, le lecteur de la BD peut s'identifier plus fortement avec les protagonistes. (...) Avec ces deux fils de la narration, un niveau élevé de dramatisation est créé. Ils se croisent, le lecteur peut se mettre alternativement dans la peau de Laurie et de Ben, il en sait du coup davantage que les protagonistes eux-mêmes sur l'histoire. La tension s'en trouve accrue ${ }^{31}$.

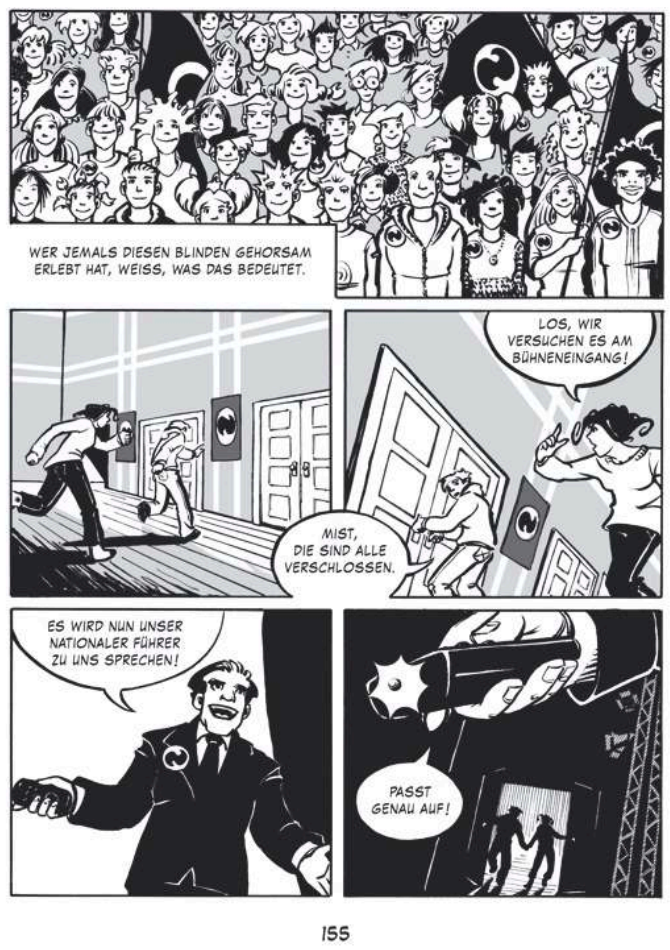

Par ailleurs, quoique fidèle à l'économie générale du roman, Kampmann a opéré quelques modifications à la marge afin de renforcer la dynamique interne du récit, parfois un peu déficiente dans l'ouvrage originel : les critiques s'accordent à dire que le livre de Rhue n'est pas, d'un point de vue littéraire, de la meilleure qualité32.

Notamment, on remarque que le découpage des chapitres a été repensé. Une césure est par exemple introduite p. 53 (ce qui correspond à la p. 61 du roman), après la première expérience de la dynamique communautaire ; le chapitre se clôt ainsi sur un crescendo, et le récit marque une pause réflexive essentielle au propos - là où Rhue reprenait le fil de la narration sans effet particulier et sans mise en valeur d'un moment pourtant décisif. Dans le même ordre d'idées, certains épisodes (tirés notamment des chapitres 11 et 12 du roman) ont été regroupés et condensés pour augmenter la fluidité du récit. Certaines autres péripéties sont reléguées à l'arrière-plan, quand elles apparaissent superflues; ainsi, il est à plusieurs reprises question, dans le roman de Rhue, du fait qu'un jeune Juif a été molesté par ses camarades. Ce fait est à peine évoqué par Kampmann qui, interrogée sur le sujet, a confirmé notre hypothèse : la judéité du 
personnage n'apporte rien à l'intrigue, et compte moins que le processus général de constitution du groupe qui crée de l'exclusion - cette exclusion est basée sur l'appartenance au mouvement, et non à une religion ${ }^{33}$.

31 Les aménagements visant à accentuer la cohérence interne de l'œuvre concernent également la caractérisation des personnages. Certains ont été carrément laissés de côté ; la femme du professeur et la mère de Laurie sont par exemple évincées du récit de Kampmann. Ils n'ont plus leur place dans un récit graphique qui vise l'efficacité et réduit le nombre d'acteurs à l'essentiel. Plusieurs personnages ont aussi vu leur nom modifié ; c'est le cas en particulier pour l'élève Deutsch, qui devient Craig, parce qu'une dénomination qui pouvait fonctionner dans le roman (américain) de Rhue crée un effet étrange pour le lecteur allemand ${ }^{34}$. Sur le plan visuel, la simplification et la clarification de la constellation des personnages vont de pair avec une forte typisation, l'essentiel étant que les acteurs "fonctionnent" pour le lecteur-spectateur dans le récit en images $^{35}$ : Éric, jeune homme aux cheveux bruns dans le roman, est chauve avec un petit bouc et des lunettes chez Kampmann - cela l'individualise par rapport à d'autres personnages bruns puisque, par la force des choses, dans une BD en noir et blanc, les cheveux sont forcément noirs (bruns) ou blonds (blancs).

Dans l'ensemble, on remarque donc que tout en respectant la trame originelle et en reprenant les personnages principaux, Kampmann introduit de légères variations, nécessaires à l'économie de sa propre œuvre; de la sorte, elle parvient à créer une dynamique spécifique qui se révèle, à maints égards, plus affirmée que celle du roman. Les moyens mis en œuvre pour « attraper » le lecteur et favoriser l'identification aux protagonistes apparaissent en définitive au moins aussi pertinents que la transpositiondramatisation réalisée par Gansel dans son film: pour Kampmann, pas besoin de « délocaliser » l'histoire dans l'Allemagne d'aujourd'hui, avec des jeunes qui taggent les façades et créent un groupe sur Myspace, ni de faire mourir ou emprisonner les protagonistes...

33 Sur un point important néanmoins, Kampmann a pris une grande liberté avec le roman de Rhue : il s'agit du moment qui succède immédiatement à l'annonce par le professeur que l'expérience est terminée, qu'il n'y a pas de grand chef du mouvement, mais simplement un notable potentiel de violence en chacun. Chez Morton Rhue, le lecteur reste un peu sur sa faim, voire assez dubitatif : après une expérience d'une grande intensité, on voit l'ex-dictateur-professeur et Robert, son plus fidèle disciple (qui vient de subir une immense déception), partir simplement déjeuner ensemble, comme si de rien n'était. Kampmann laisse au contraire "Mister Ross " seul en scène, devant des tribunes vides, sans la scène de réconciliation qui sonne un peu faux. Elle ajoute ensuite un épilogue sur la vie qui reprend son cours, malgré le choc qui laisse des traces; d'un point de vue visuel comme dramatique, la boucle est bouclée avec le tout début du récit, puisque la $\mathrm{BD}$ s'ouvrait sur la ville paisible avant la tempête. L'auteure confère à l'ensemble une unité qui est absente dans le roman.

Désamorçant, par différentes techniques, le risque d'une «trivialisation » du sujet et exploitant le potentiel émotionnel particulier du medium BD, Stefani Kampman est parvenue à créer une œuvre autonome, qui modernise la matière originelle. Contournant l'écueil d'une démarche didactique lourde, elle semble avoir trouvé un 
juste équilibre entre la nécessité d'attirer et divertir un public spécifique, et l'obligation de se tenir au ton sérieux qui convient au sujet.

Un petit clin d'œil au lecteur, dans l'une des dernières pages du roman graphique, vient pourtant rappeler à quel point la reconnaissance de la bande dessinée est fragile et toujours potentiellement mise en doute: Robert, le marginal pataud qui avait trouvé une formidable occasion d'intégration grâce à Die Welle, retourne après la fin de l'expérience à ses distractions de mauvais élève, c'est-à-dire... qu'il lit un comix ! (ill. 6)

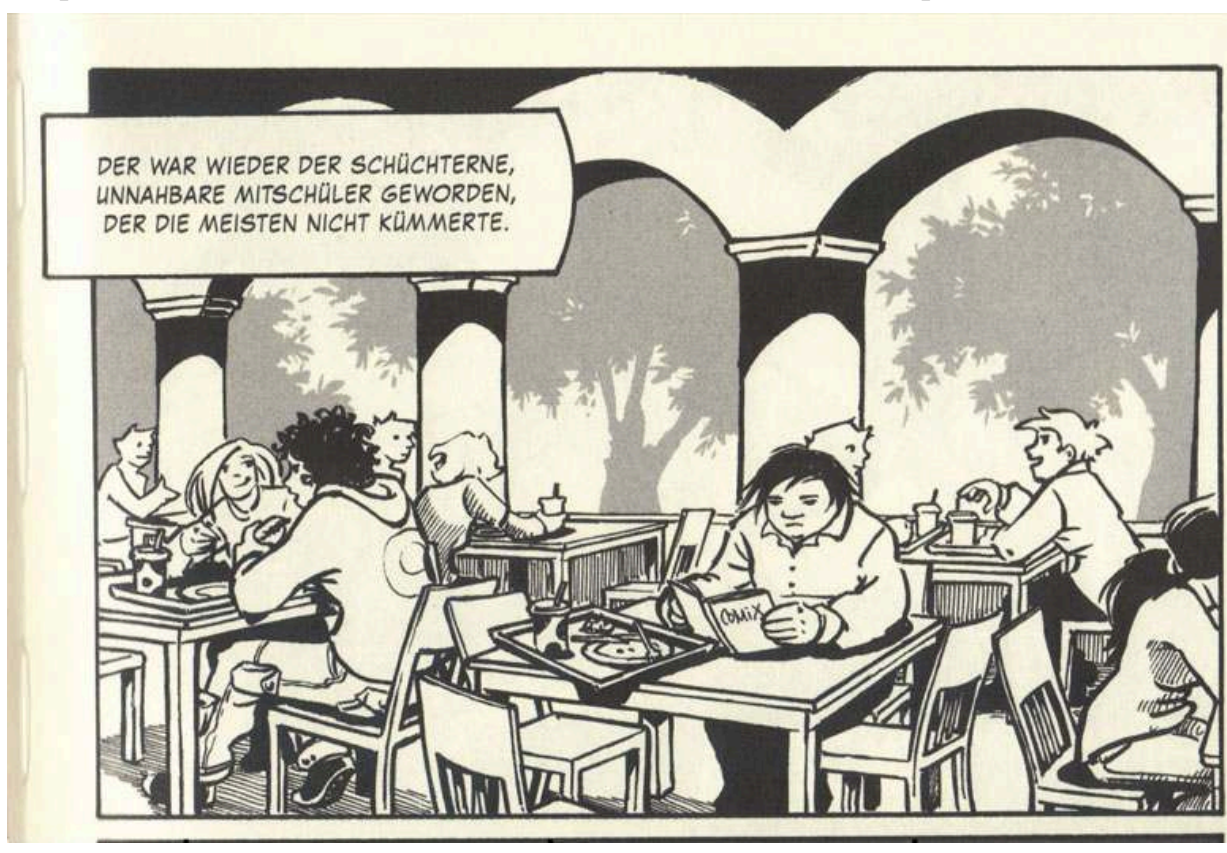

Cette anecdote apparemment tout à fait secondaire - au point qu'elle échappe facilement au regard - peut être comprise comme une interrogation persistante, au cœur même d'un ouvrage qui semble pourtant y apporter un fort démenti : la bande dessinée resterait-elle un genre mineur, réservé aux cancres?

\section{NOTES}

1. Sous-titre programmatique sur la page de titre de l'ouvrage de Kerstin Winter: Die Welle. Roman nach dem Film von Dennis Gansel, Ravensburger Buchverlag, Ravensburg, 2008.

2. Die Welle. Ein Film von Dennis Gansel, Constantin Filmproduktion, 2008.

3. Stefani Kampann : Die Welle. Eine Graphic Novel nach dem Roman von Morton Rhue, Ravensburger Buchverlag, Ravensburg, 2007.

4. Voir le récit de l'expérience dans l'article «"Third Wave" presents inside look into Fascism », in: The Catamount. Cubberly High-school, volume 11, n¹4, 21 avril 1967, p. 3. Je tiens ici à remercier Anastasia Marcante, étudiante de Master I en 2008-2009, pour les articles de presse qu'elle a collectés et les interviews qu'elle a réalisées et mis à ma disposition suite à ses propres recherches sur le film Die Welle. 
5. Stanley Milgram : Das Milgram-Experiment. Zur Gehorsamsbereitschaft gegenüber Autorität, Rowohlt, Reinbek /Hambourg, 1982.

6. In :récit de Jones intitulé The Third Wave, accessible sur la page Internet de Todd Strasser (alias Morton Rhue) : http://www.toddstrasser.com/html/thewave2.htm

7. L'adaptation de Die Welle a été réalisée pour la chaîne de télévision américaine $A B C$ par Alexander Grasshoff (The Wave, TAT Communication Company, Tandem Productions, 1981).

8. Cf. courrier de Kampmann à l'auteure du présent article, le 17 juillet 2010. Tous mes remerciements à Mme Kampmann pour la précision de ses réponses dans les échanges de lettres qui ont eu lieu entre mai et août 2010.

9. Kerstin Winter, op. cit.

10. Blog consacré à la bande dessinée : http://comicsmarvel.blogspot.com/2009/04/die-welle-lepouvoir-par-la-discipline.html (article « Le pouvoir par la discipline » signé Neault, publié le 27 avril 2009)

11. Voir, entre autres, l'introduction d'Etienne François, Hagen Schulze (dir.): Deutsche Erinnerungsorte, Beck,Munich, 2001; Aleida Assmann, Ute Frevert: Geschichtsvergessenheit Geschichtsversessenheit. Vom Umgang mit deutschen Vergangenheiten nach 1945, Deutsche VerlagsAnstalt (DVA), Stuttgart 1999.

12. Cf. l'interview de Dennis Gansel dans le journal de cinéma Première, consultable sur la page : http://www.premiere.fr/Cinema/Exclus-cinema/Interview-cinema/La-vague-interview-deDennis-Gansel/(gid)/ 1531011

13. Cf. Maximilian Probst: «Macht durch Handeln! Dennis Gansels Verfilmung des Bestsellerromans Die Welle überträgt die Verführungskraft des Faschismus überzeugend auf die Gegenwart ", in : Die Zeit, 13.03.2008.

14. Alexandra Oeser : Enseigner Hitler. Les adolescents face au passé nazi en Allemagne, Éditions de la Maison des sciences de l'homme, Paris, 2010.

15. Sur les discours à propos du genre de la bande dessinée, voir Dusquier et Pabreuil : «Du Voyou au Critique : parler de la Bande Dessinée », p. 11-60 in : revue Labyrinthe, 2006 (3), n²5, n thématique sur La Bande dessinée. Ce qu'elle dit, ce qu'elle montre, coordonné par Laurent Dubreuil, Renaud Pasquier et Guillaume Paugam.

16. Pierre Alban Delannoy: Maus d'Art Spiegelmann: Bande dessinée et Shoah, Champs visuels, L'Harmattan, Paris, 2002, p. 10. L'auteur fait ici référence à l'article de Robert S. Levanthal : «Art Spiegelmann's MAUS: Working-Through the Trauma ot the Holocaust », publié en 1995 sur le site jefferson.village.virginia.edu/holocaust/spiegelmann.

17. Voir le recueil de contributions universitaires: Odette Mitterrand, Gilles Ciment (dir.): L'Histoire... par la bande. Bande dessinée, histoire et pédagogie, Syros, Paris 1993. Voir également la constitution récente de groupe de travail consacré aux « circulations, transferts et adaptations dans la bande dessinée» au Centre d'histoire culturelle des sociétés contemporaines de l'Université de Versailles Saint-Quentin.

18. Voir Marc Hieronimus : "Geschichtscomics im DaF-Unterricht », in : Magali Laure Nieradka, Denise Specht (dir.) : Fremdkörper? Aspekte der Geisteswissenschaften in der Auslandsgermanistik und im DaF-Unterricht, Lit, Berlin, 2009, p. 101-114.

19. Blog consulté le 5 juin 2010: http://www.hr-online.de/website/specials/buchmesse2009/ index.jsp?rubrik=38546\&key=standard_rezension_35397838

20. Doris König : «Die visuelle Sprache des Schreckens : Stefani Kampmanns Graphic Novel, Die Welle (2007) nach Morton Rhue ", www.rezensionsprofis.de, Kinder \& Jugend, 29.09.2008, http:// www.rezensionsprofis.de/kinder-jugend-03-stefani-kampmanns.html (consulté le 7 juin 2010).

21. «Eine Graphische Novelle vom Unterwerfen, Warum Hitler Erfolg hatte», article signé RA http://www.schenken.net/cgi-bin/db_site.cgi/site_1866/ (consulté le 9 juin 2010). 
22. Birte Ziemann Freitag: «Die Welle - Eine Graphic Novel», 16 Mai 2008, http:// www.literaturnetz.com/index.php?/Buch/Comic/Die-Welle-Eine-Graphic-Novel.html (consulté le 9 juin 2010). Voir aussi Doris König, op. cit.

23. Stefani Kampmann, courrier du 13 août 2010.

24. Stefani Kampmann, courrier du 17 juillet 2010.

25. Jones a même fait office de « consultant » pour le film de Gansel.

26. Cf. Cristina Nord : «Deutsche Geschichte im Kino. Die neue Naivität», in : taz, 20 octobre 2008. Des considérations utiles sur le sujet également in : Marc Hieronimus, op. cit., p. 112-113.

27. Cf. Roland Barthes : La chambre claire, Cahiers du cinéma/Gallimard, Paris 1980.

28. Voir sur ce sujet les réflexions essentielles de Pascal Lefèvre : «Projections : l'écran cité », in : Gilles Ciment (dir.) : Cinéma et bande dessinée, hors-série de CinémAction, Corlet-Télérama, Condé, 1990.

29. Notamment avec la modification de la fin de l'histoire : le film s'achève en effet sur le suicide d'un élève et l'arrestation du professeur. Cette dramatisation n'a pas fait l'unanimité, en raison de son caractère spectaculaire et presque caricatural, voir par exemple Rüdiger Suchsland : «Club der toten Nazis. 'Ich weiss nicht, ob ich da nicht auch' ne Glatze geworden wäre», in : Arteschock, sans date, consultable sur: http://www.arteschock.de/film/text/kritik/w/welle.htm.

30. Cf. Gansel interviewé par Der Standart, 12 février 2008 ; voir aussi Hanns-Georg Rodek: «Wieviel Nazi steckt in jedem Kind?», in : Die Welt, 11 mars 2008.

31. Stefani Kampmann, courrier du 17 juillet 2010.

32. Ainsi, le critique de la Süddeutsche Zeitung disait au moment de la sortie du film: «ce qui plaidait contre un film à partir du roman Die Welle, c'était (...) en premier lieu le livre lui-même. Son succès est immense, mais c'est aussi un livre indiciblement mauvais - une sorte de Oui-Oui contre les faschistes - et cette comparaison risque de faire du tort à Enid Blyton », Tobias Kniebe : « Der Faschist in uns », 07.09.2009.

33. Stefani Kampmann, courrier du 13 août 2010.

34. Ibid.

35. Selon l'expression de Stefani Kampman dans son courrier du 17 juillet 2010.

\section{RÉSUMÉS}

Parallèlement à son adaptation en film, le roman à succès de Morton Rhue sur le nazisme a récemment été mis en images dans une bande dessinée au graphisme proche de l'univers manga. Désamorçant par différentes techniques le risque d'une «trivialisation » du sujet et exploitant le potentiel émotionnel particulier du medium BD, l'auteure est parvenue à créer une œuvre qui modernise la matière originelle et peut ainsi toucher un large public. La comparaison avec la démarche du cinéaste fait ressortir les particularités de chaque support et met en lumière les moyens spécifiques dont dispose la bande dessinée. Après la présentation des différents avatars de Die Welle - de la réalité d'une expérience aux diverses formes de récits (romanesques, filmiques, dessinés), on examine plus précisément les stratégies de légitimation et les procédés concrets d'adaptation mis en œuvre par Kampmann dans son « roman graphique ».

Gleichzeitig mit seiner Verfilmung wurde der Bestseller-Roman von Morton Rhue über den Nationalsozialismus jüngst auch zu einer am Manga-Stil orientierten Graphic Novel. Die Autorin wendet Tricks an, um dem Vorwurf einer «Trivialisierung » bzw. Verharmlosung des Themas 
vorzubeugen; dadurch, dass sie sich das emotionale Potential des Mediums "Comic» am Geschicktesten zunutze macht, ist es ihr gelungen, ein Werk $\mathrm{zu}$ schaffen, welches das ursprüngliche Material modernisiert und ein breites Publikum zu erreichen vermag. Der Vergleich mit dem Ansatz des Filmemachers hebt die Besonderheiten des jeweiligen Mediums hervor und bringt die spezifischen Möglichkeiten der Comics zur Geltung. Nach der Präsentation der verschiedenen Avatare von Die Welle - vom "wirklichen» Experiment bis zu den verschiedenen Formen von Erzählungen (im Roman, Film und Comic) - werden die in Graphic Novel von Kampmann eingesetzten Legitimierungsstrategien und konkreten Adaptionsverfahren untersucht..

Along side a film adaptation, the bestselling novel by Morton Rhue about Nazism has recently been made into a cartoon that is closed to the manga universe. The author has managed to create a work that modernises the original material and can reach a wide audience. This is achieved through the prevention of «trivialisation » of the subject matter by various techniques, and the exploitation of the emotional potential of the graphic novel medium. The comparison with the filmmaker's approach highlights the peculiarities of each medium and the specific resources available to comics. After the presentation of the various avatars of Die Welle - from the reality of an experience to the various forms of storytelling (in novels, films, comics) -, we examine in depth the legitimising strategies and concrete adaptation processes that Kampmann implements in her « graphic novel».

\section{AUTEURS}

\section{CLAIRE ASLANGUL}

Université Paris IV 